\title{
How I Do It
}

Timothy Carman, MD, FISHRS La Jolla, California, USA tcarmanmd@ljhr.com

\section{Donor Closures, Tables, Potato Chip Bags, and All}

In this issue of How I Do It, I want to offer small, but I think significant, details associated with my current preference in donor closure technique. Quite a lot of discussion can go into the subject: single layer, double layer, sutures vs. staples, absorbable vs. non-absorbable, trichophytic vs. non-trichophytic, etc., etc.

I will outline the basis of the majority of my closures, and the rationale for my approach. Again, this is just my opinion, nothing more. I am of the view that what "works" in a given surgeon's hands is what is "best" for his or her patients.

There are several principles to be observed when talking about donor closures in general. My overriding principle is to respect the tissue. This implies making appropriate judgment calls regarding the amount of tissue that can be safely removed without exposing the wound closure to unnecessary tension. This requires an accurate assessment of laxity as well as locating the strip in the center of the "safe zone" (please see How I Do It: The "Sweet Spot" for Strip Harvesting in the January/February 2014 issue of the Forum; 24(1):14).

Pre-surgery scalp stretching exercises can improve laxity. Assuming this initial judgment call is correct, the issue of requiring a separate, "stronger," deep layer of sutures becomes moot, which leads to my first observation in practice: most donor wounds are best closed utilizing a single layer running suture. The reasoning behind the running suture is simple: having a single thread along the donor closure line provides equal tension all along the incision, so that no one area has more pressure between the skin edges as opposed to other areas. The idea is to allow blood flow, not to constrict it. Equally important is the distance of no more than $3-4 \mathrm{~mm}$ between the loops of suture. The principle here again is related to prevention of constriction of blood flow in the tissue, and to reducing the "tension" each suture holds. The more throws per linear length, the less tension each must bear. The analogy would be two individuals versus four lifting a table. With two individuals lifting a 100-pound table, there will be 25 pounds per arm distributed weight. With four individuals lifting the table, that amount decreases to 12.5 pounds per arm. This concept is also one of the reasons I prefer not to utilize the "two-layer" closure technique. Most of the time, "deep layer" suture bites are taken at wide intervals, exacerbating the amount of focal tension on the tissue where they are "anchored," and consequently this can negatively affect local blood flow. They are also typically placed a greater distance from the incision edges than where the "superficial layer" skin sutures are placed, so an even greater area of tissue is

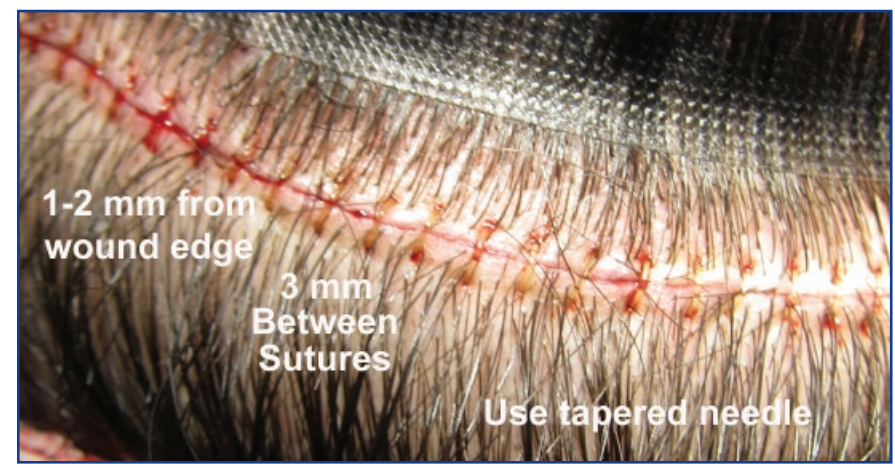

Figure 1. Close-up detail of closure technique. unevenly exposed to intermittent increases in tension in the closure.

Of equal importance is the distance from the skin edges that the sutures are placed. This distance should normally be $1-2 \mathrm{~mm}$ at most. It is also important to observe the rule "deeper than wide" in that the suture should course between the two edges across the wound at a depth of $3-4 \mathrm{~mm}$.

The single greatest impediment to performing the closure I found is the type of needle used. Most skin suture needles are some form of cutting needle. I have found that these, when applied specifically to donor closures in hair transplantation, can be counterproductive. The best analogy for my observation I call the "Potato Chip Bag" opening phenomena. Think of the material most potato chip bags are made of. You can pull on that bag every which way and the material won't tear. What is the next move? That's right-typically one takes a small "bite" out of the bag. That's usually all it takes; the bag will then tear, almost too easily. This same principle occurs when using a cutting needle. My solution has been for years to use a tapered needle instead. This action "punctures" the skin on entry and exit, but does not produce any "starting edges" along which the skin may tear. This allows me to take bites closer to the wound edge, and accordingly lessens the amount of tissue subjected to constriction by the suture.

Last, but not least, is the question of trichophytic closure. In general, I am selective in its use. I am less likely to use this closure on a patient with very straight hair, as I have noticed (while in line at Starbucks, actually, standing behind a hair transplant patient) that even with hair that is long enough to cover the scar, there can be a "line" in the patients hair that parallels the incision. This comes about by the change in angulation that may occur to the hairs along the border that has the epidermis removed in the trichophytic technique. Consequently, this telltale sign is the exact opposite of the intention of utilizing the technique. While a trichophytic closure may succeed at filling in the scar somewhat with hair, the price in these patients is a visible "demarcation line." This problem is mitigated in patients that have a wave or slight curl to their hair, and these are patients I am more apt to use the trichophytic option on, although, I must say, in general, by using the donor closure technique outlined above I have managed to avoid the indication that the trichophytic closure was intended for initially in the first place.

Just my two cents.

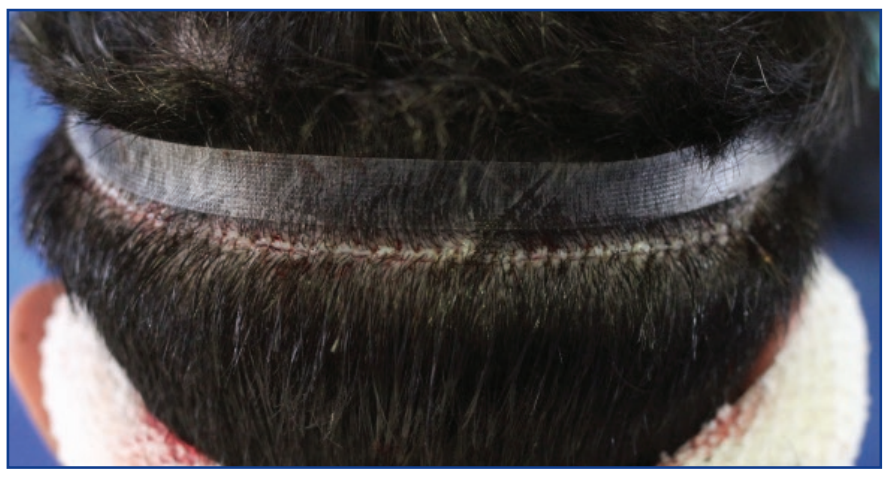

Figure 2. Typical closure. 